\title{
Doppler images of II Pegasi for 2004-2010^
}

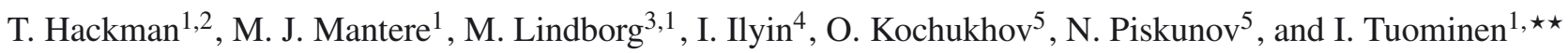 \\ 1 Department of Physics, PO Box 64, 00014 University of Helsinki, Finland \\ e-mail: Thomas. Hackman@helsinki . fi \\ 2 Finnish Centre for Astronomy with ESO, University of Turku, Väisäläntie 20, 21500 Piikkiö, Finland \\ 3 Nordic Optical Telescope, 38700 Santa Cruz de la Palma, Spain \\ ${ }^{4}$ Leibniz-Institut für Astrophysik Potsdam, An der Sternwarte 16, 14882 Potsdam, Germany \\ 5 Department of Physics and Astronomy, Uppsala University, Box 516, 75120 Uppsala, Sweden
}

Received 30 June 2011 / Accepted 15 December 2011

\section{ABSTRACT}

\begin{abstract}
Aims. We study the spot activity of II Peg during the years 2004-2010 to determine long- and short-term changes in the magnetic activity. In a previous study, we detected a persistent active longitude, as well as major changes in the spot configuration occurring on a timescale of shorter than a year. The main objective of this study is to determine whether the same phenomena persist in the star during these six years of spectroscopic monitoring.

Methods. The observations were collected with the high-resolution SOFIN spectrograph at the Nordic Optical Telescope. The temperature maps were calculated using a Doppler imaging code based on Tikhonov regularization.

Results. We present 12 new temperature maps that show spots distributed mainly over high and intermediate latitudes. In each image, 1-3 main active regions can be identified. The activity level of the star is clearly lower than during our previous study for the years 1994-2002. In contrast to the previous observations, we detect no clear drift of the active regions with respect to the rotation of the star.

Conclusions. Having shown a systematic longitudinal drift of the spot-generating mechanism during 1994-2002, the star has clearly switched to a low-activity state for 2004-2010, during which the spot locations appear more random over phase space. It could be that the star is near to a minimum of its activity cycle.
\end{abstract}

Key words. stars: imaging - stars: activity - starspots - stars: individual: HD 224085

\section{Introduction}

II Peg (HD 224085) is an extremely active RS CVn star, exhibiting both strong chromospheric and coronal activity, in addition to photometric variability caused by spots. It has had one of the highest observed photometric amplitudes (Doyle et al. 1989) and is also claimed to be the brightest X-ray source within $50 \mathrm{pc}$ (Makarov 2003). More recent studies of II Peg include estimates of its differential rotation using ground-based (Roettenbacher et al. 2011) and satellite observations (Siwak et al. 2010). Both studies indicate a weak solar-type differential rotation. The latter study based on data from the MOST satellite confirmed previous findings that flares must be related to active regions, since they are more frequently observed when the most spotted hemisphere is visible (e.g. Mohin \& Raveendran 1993; Teriaca \& Catalano 1999; Frasca et al. 2008).

II Peg has been spectroscopically monitored for nearly 20 years with the SOFIN spectrograph at the Nordic Optical Telescope (NOT, La Palma, Spain). Its observations have enabled both Doppler imaging and long-term studies of the spot activity to be performed (Berdyugina et al. 1998a, 1999; Lindborg et al. 2011). A summary of these results can be found in our earlier paper Lindborg et al. (2011), which was based on observations from 1994 to 2002. In these Doppler images, there

\footnotetext{
* Based on observations made with the Nordic Optical Telescope, operated on the island of La Palma jointly by Denmark, Finland, Iceland, Norway, and Sweden, in the Spanish Observatorio del Roque de los Muchachos of the Instituto de Astrofisica de Canarias.

$\star \star$ Deceased.
}

were usually two active longitudes, one of which was persistent and stronger almost throughout the nine years of spectroscopic monitoring. Major changes, occurring on a timescale of shorter than one year, could also be seen. For instance, the spot activity could switch to another, weaker, longitude, for a short period of time. These shifts are similar to the "flip-flops" described by Berdyugina and collaborators (Berdyugina et al. 1998a; Berdyugina \& Tuominen 1998; Berdyugina et al. 1999), except that they were not found to follow any periodicity. In addition to the short-term shifts of the active longitudes, we also discovered a drift in the active regions with respect to the orbital rotation frame, indicating that the spot-generating structure rotated slightly faster than the tidally locked binary system.

Rapidly rotating late-type stars with deep convective envelopes are expected to exhibit very little differential rotation, because it is theoretically predicted to be suppressed in the rapid rotation regime (e.g. Kitchatinov \& Rüdiger 1999); for II Peg, this has also been observationally confirmed (Siwak et al. 2010; Roettenbacher et al. 2011). However, several stars have been found to contradict this prediction (e.g. Hackman et al. 2001; Jeffers \& Donati 2008; Frasca et al. 2011). Furthermore it is unclear whether surface differential rotation can be recovered by following the motion of large spots (Korhonen \& Elstner 2011). Nevertheless, in the case of suppressed differential rotation, the dynamos working in rapidly rotating stars are expected to be of the $\alpha^{2}$-type, the magnetic field generation only being due to the inductive action of convective turbulence. According to both linear (e.g. Krause \& Rädler 1980) and nonlinear solutions (e.g. Moss et al. 1995) of the $\alpha^{2}$-dynamo equations, the 
nonaxisymmetric modes become more easily excited in the rapid rotation regime. The $m=1$ mode, representing an azimuthally varying field changing sign once over the full longitude span, is commonly the preferred field configuration. This is unsurprising, as the largest scale mode is always the least affected by diffusive effects.

The nonaxisymmetric modes turn out to be waves migrating in the azimuthal direction, not necessarily having the rotation period of the star (e.g. Krause \& Rädler 1980). Both slower and faster dynamo waves can occur, depending for example on the profile and properties of the turbulent transport coefficients. The faster waves with dipole symmetry (S1) were found to be preferred in linear models with simple profiles (Krause \& Rädler 1980), and slower waves with quadrupolar symmetry (A1) in more complicated nonlinear models when solving for the dynamics (Tuominen et al. 2002). From the viewpoint of dynamo theory, two migratory active longitudes are thus an expected result. We, therefore, interpret the behaviour seen in II Peg during 1994-2002 as a manifestation of such a dynamo wave. The objective of the present paper was to inspect whether the azimuthal dynamo wave persisted on the star in the most recent observational data of the object.

\section{Observations}

Our observations were made using the SOFIN high-resolution échelle spectrograph at the $2.56 \mathrm{~m}$ NOT, La Palma, Spain. The data were acquired with the second camera equipped with a Loral CCD detector with $2048 \times 2048$ pixels. This provides a spectral resolution of $R \approx 70000$.

A total of 12 sets of high-resolution spectra of II Peg were measured in 2004-2010. The signal-to-noise ratio $(\mathrm{S} / \mathrm{N})$ of the observations was usually around 200-300. The number of observed phases ranged from 3 to 12 .

In general, observations of about ten evenly distributed rotation phases is considered optimal for Doppler imaging (e.g. Vogt et al. 1987). The spatial resolution of the Doppler imaging depends on the phase coverage, but the phase coverage is more important for determining the right spot latitude than the longitude. In the case of gaps in the observations, the reliability of the spot positions depends on the visibility of the spots at the times of observations. This means that observations with insufficient phase coverage can still be very useful when studying e.g. active longitudes. We estimated the fraction $f$ of the rotation phases covered by the observations by assuming that each observation covers \pm 0.05 of the rotation period. Thus, ten observations uniformly distributed in phase would give a $100 \%$ coverage.

The spectral regions 5392.3-5395.1 $\mathrm{A}, 5524.7-5527.3 \AA$, and 5633.2-5634.6 $\AA$ were chosen for Doppler imaging. The regions contain relatively unblended lines of different atoms, ionization states, and strengths. Owing to the diversity of the line parameters, the Doppler imaging solution becomes more reliable.

The ephemeris used to calculate the phases

$T_{\text {conj }}=2449582.9268+6.724333 E$

was taken from Berdyugina et al. (1998a). A summary of the observations is given in Table 1. A more complete listing of the heliocentric Julian dates, phases calculated according to the ephemeris given above, and the $\mathrm{S} / \mathrm{N}$ of each observation is given in Table 2.

The spectral observations were reduced with the 4A software system (Ilyin 2000). Bias, cosmic ray, flat-field and scatteredlight corrections, wavelength calibration and normalization, and
Table 1. Summary of observations with $\mathrm{NOT}^{a}$.

\begin{tabular}{lcccccc}
\hline \hline Season & $t_{\min }$ & $t_{\max }$ & $\mathrm{S} / \mathrm{N}$ & $n_{\phi}$ & $f_{\phi}$ & $\begin{array}{c}d \\
\times 10^{-3}\end{array}$ \\
\hline Aug. 04 & 53216.6 & 53228.6 & 244 & 8 & $75 \%$ & 5.70 \\
Dec. 04 & 53370.4 & 53372.4 & 177 & 3 & $30 \%$ & 6.19 \\
Jul. 05 & 53567.7 & 53575.6 & 218 & 7 & $56 \%$ & 5.94 \\
Nov. 05 & 53685.5 & 53695.5 & 197 & 5 & $38 \%$ & 6.73 \\
Sep. 06 & 53978.5 & 53992.7 & 259 & 11 & $72 \%$ & 6.10 \\
Dec. 06 & 54071.5 & 54078.4 & 166 & 7 & $63 \%$ & 6.66 \\
Jul. 07 & 54300.7 & 54309.7 & 264 & 10 & $81 \%$ & 5.84 \\
Nov. 07 & 54427.4 & 54437.4 & 238 & 7 & $63 \%$ & 6.19 \\
Sep. 08 & 54717.6 & 54723.5 & 304 & 4 & $40 \%$ & 5.37 \\
Dec. 08 & 54809.4 & 54815.5 & 263 & 6 & $59 \%$ & 7.19 \\
Aug. 09 & 55069.7 & 55081.7 & 291 & 12 & $87 \%$ & 6.82 \\
Dec. 09 & 55193.4 & 55201.4 & 283 & 8 & $68 \%$ & 6.02 \\
\hline
\end{tabular}

Notes. ${ }^{(a)}$ Season, HJD -2400000 of first $\left(t_{\min }\right)$ and last $\left(t_{\max }\right)$ observation, mean $\mathrm{S} / \mathrm{N}$, number of observed phases $\left(n_{\phi}\right)$, estimated phase coverage $\left(f_{\phi}\right)$, and deviation $(d)$ of the Doppler imaging solution.

corrections for the motion of the Earth as well as the orbital motion of the binary system were included in the reduction process. For the orbital motion, we used the solution presented by Berdyugina et al. (1998b). The continuum normalization was done in two steps. The spectral orders were first normalized by a polynomial continuum fit of third degree. In rapidly rotating stars, lines are blended, and there may not be any real continuum within a spectral interval. Therefore, an additional continuum correction for each wavelength interval used for Doppler imaging was done by comparing the seasonal average observed profile and a synthetic line profile. Near-continuum points were used for a first or second degree polynomial fit to correct the normalized flux level.

\section{Doppler imaging}

The same Doppler imaging method as that applied in Lindborg et al. (2011) was used to calculate temperature maps for II Peg. The inversion technique was thoroughly described in several papers (Piskunov 1991; Hackman et al. 2001; Lindborg et al. 2011). The main difference with our previous analysis was that we used the new MARCS model atmospheres (Gustafsson et al. 2008) for the line profile calculations.

\subsection{Stellar and spectral parameters}

Several different sets of stellar parameters have previously been suggested for II Peg. The choice of parameters is far from trivial in the sense that different parameters have very similar effects on the spectral lines and good fits can be obtained using different combinations of stellar and spectral parameters. For example, changing the value of the microturbulence will cause shifts in the average effective temperature $T_{\text {eff }}$ of the solution. However, the effect of the spots on the surface is, up to first order, the same independent of the chosen set of parameters: cool spots cause "emission bumps" in the photospheric spectral lines.

For the rotational velocity, macroturbulence, rotation period, and inclination angle, we used the values adopted by Berdyugina et al. (1998a). An unspotted star with $T_{\text {eff }}=4750$ was used as an initial guess of the surface temperature. Other parameters were chosen by comparing the mean seasonal observations with synthetic spectra of this unspotted star. In practice, the parameters 
Table 2. Observations with $\mathrm{NOT}^{b}$.

\begin{tabular}{|c|c|c|c|c|c|c|c|c|c|c|c|}
\hline $\begin{array}{l}\text { Date } \\
\text { (dd/mm/yyyy) }\end{array}$ & $\overline{\mathrm{HJD}}$ & $\overline{\phi \phi}$ & $\overline{S / N}$ & $\begin{array}{c}\text { Date } \\
\text { (dd/mm/yyyy) }\end{array}$ & $\overline{\mathrm{HJD}}$ & $\overline{\phi \phi}$ & $\overline{\mathrm{S} / \mathrm{N}}$ & $\begin{array}{c}\text { Date } \\
\text { (dd/mm/yyyy) }\end{array}$ & $\overline{\mathrm{HJD}}$ & $\bar{\phi}$ & $\overline{\mathrm{S} / \mathrm{N}}$ \\
\hline $30 / 07 / 2004$ & 3216.618 & 0.379 & 190 & $11 / 09 / 2006$ & 3989.704 & 0.348 & 314 & $11 / 09 / 2008$ & 4720.555 & 0.035 & 290 \\
\hline $31 / 07 / 2004$ & 3217.646 & 0.532 & 291 & $12 / 09 / 2006$ & 3990.649 & 0.488 & 404 & $13 / 09 / 2008$ & 4722.626 & 0.343 & 320 \\
\hline 01/08/2004 & 3218.648 & 0.681 & 249 & $13 / 09 / 2006$ & 3991.680 & 0.642 & 169 & $14 / 09 / 2008$ & 4723.544 & 0.480 & 306 \\
\hline 02/08/2004 & 3219.644 & 0.829 & 247 & $14 / 09 / 2006$ & 3992.680 & 0.790 & 213 & $08 / 12 / 2008$ & 4809.393 & 0.247 & 383 \\
\hline 03/08/2004 & 3220.653 & 0.979 & 201 & $01 / 12 / 2006$ & 4071.466 & 0.507 & 198 & $09 / 12 / 2008$ & 4810.421 & 0.399 & 104 \\
\hline $05 / 08 / 2004$ & 3222.612 & 0.271 & 227 & $03 / 12 / 2006$ & 4072.526 & 0.665 & 149 & $10 / 12 / 2008$ & 4811.478 & 0.557 & 222 \\
\hline $10 / 08 / 2004$ & 3227.721 & 0.031 & 270 & $04 / 12 / 2006$ & 4074.413 & 0.945 & 183 & $11 / 12 / 2008$ & 4812.439 & 0.700 & 260 \\
\hline $11 / 08 / 2004$ & 3228.615 & 0.164 & 278 & $05 / 12 / 2006$ & 4075.472 & 0.103 & 159 & $13 / 12 / 2008$ & 4814.437 & 0.997 & 340 \\
\hline $30 / 12 / 2004$ & 3370.395 & 0.248 & 237 & $06 / 12 / 2006$ & 4076.442 & 0.247 & 188 & $14 / 12 / 2008$ & 4815.474 & 0.151 & 268 \\
\hline $31 / 12 / 2004$ & 3371.377 & 0.394 & 192 & $07 / 12 / 2006$ & 4077.427 & 0.394 & 151 & 26/08/2009 & 5069.676 & 0.954 & 302 \\
\hline 01/01/2005 & 3372.382 & 0.544 & 103 & $08 / 12 / 2006$ & 4078.415 & 0.540 & 136 & $27 / 08 / 2009$ & 5070.687 & 0.105 & 290 \\
\hline $16 / 07 / 2005$ & 3567.704 & 0.591 & 210 & 19/07/2007 & 4300.696 & 0.597 & 233 & 28/08/2009 & 5071.725 & 0.259 & 184 \\
\hline $17 / 07 / 2005$ & 3568.713 & 0.741 & 205 & 20/07/2007 & 4301.710 & 0.747 & 266 & 29/08/2009 & 5072.697 & 0.404 & 320 \\
\hline $18 / 07 / 2005$ & 3569.728 & 0.892 & 207 & $21 / 07 / 2007$ & 4302.674 & 0.891 & 276 & $30 / 08 / 2009$ & 5073.708 & 0.554 & 186 \\
\hline $19 / 07 / 2005$ & 3570.644 & 0.028 & 228 & $22 / 07 / 2007$ & 4303.630 & 0.033 & 268 & $01 / 09 / 2009$ & 5075.557 & 0.829 & 254 \\
\hline $20 / 07 / 2005$ & 3571.661 & 0.179 & 234 & $23 / 07 / 2007$ & 4304.673 & 0.188 & 242 & 02/09/2009 & 5076.682 & 0.996 & 364 \\
\hline $23 / 07 / 2005$ & 3574.675 & 0.627 & 217 & $24 / 07 / 2007$ & 4305.705 & 0.341 & 299 & 03/09/2009 & 5077.634 & 0.138 & 411 \\
\hline $24 / 07 / 2005$ & 3575.649 & 0.772 & 230 & $25 / 07 / 2007$ & 4306.643 & 0.481 & 314 & 04/09/2009 & 5078.743 & 0.303 & 333 \\
\hline $11 / 11 / 2005$ & 3685.505 & 0.109 & 122 & $26 / 07 / 2007$ & 4307.705 & 0.639 & 244 & 05/09/2009 & 5079.676 & 0.442 & 346 \\
\hline $11 / 11 / 2005$ & 3686.479 & 0.254 & 146 & $27 / 07 / 2007$ & 4308.649 & 0.779 & 275 & 06/09/2009 & 5080.672 & 0.590 & 348 \\
\hline $17 / 11 / 2005$ & 3692.484 & 0.147 & 265 & $28 / 07 / 2007$ & 4309.698 & 0.935 & 222 & 07/09/2009 & 5081.740 & 0.748 & 164 \\
\hline $19 / 11 / 2005$ & 3693.548 & 0.305 & 226 & $22 / 11 / 2007$ & 4427.437 & 0.445 & 241 & $27 / 12 / 2009$ & 5193.360 & 0.348 & 271 \\
\hline $20 / 11 / 2005$ & 3695.471 & 0.591 & 224 & $25 / 11 / 2007$ & 4430.472 & 0.896 & 152 & 28/12/2009 & 5194.381 & 0.500 & 264 \\
\hline $30 / 08 / 2006$ & 3978.502 & 0.682 & 214 & $26 / 11 / 2007$ & 4431.475 & 0.045 & 236 & $29 / 12 / 2009$ & 5195.371 & 0.647 & 366 \\
\hline 01/09/2006 & 3979.654 & 0.853 & 244 & $27 / 11 / 2007$ & 4432.454 & 0.191 & 343 & $31 / 12 / 2009$ & 5197.399 & 0.949 & 366 \\
\hline 02/09/2006 & 3980.686 & 0.007 & 240 & $30 / 11 / 2007$ & 4435.317 & 0.617 & 115 & $01 / 01 / 2010$ & 5198.411 & 0.099 & 310 \\
\hline 05/09/2006 & 3983.672 & 0.451 & 156 & $01 / 12 / 2007$ & 4436.470 & 0.788 & 294 & 02/01/2010 & 5199.376 & 0.243 & 259 \\
\hline 06/09/2006 & 3984.731 & 0.608 & 212 & $02 / 12 / 2007$ & 4437.448 & 0.934 & 290 & 03/01/2010 & 5200.363 & 0.389 & 213 \\
\hline 07/09/2006 & 3985.676 & 0.749 & 340 & 08/09/2008 & 4717.606 & 0.597 & 303 & $04 / 01 / 2010$ & 5201.385 & 0.541 & 217 \\
\hline 09/09/2006 & 3987.705 & 0.051 & 338 & & & & & & & & \\
\hline
\end{tabular}

Notes. ${ }^{(b)}$ The heliocentric Julian date is given as HJD-2 450000 and the S/N is for a wavelength region centred on $5630 \AA$.

Table 3. Stellar parameters.

\begin{tabular}{lc}
\hline \hline Parameter & Value \\
\hline Gravity & $\log g=3.5$ (in cgs-units) \\
Inclination & $i=60^{\circ}$ \\
Rotation velocity & $v \sin i=22.6 \mathrm{~km} \mathrm{~s}^{-1}$ \\
Rotation period & $P=6.724333$ \\
Metallicity & $\log [M / H]=-0.25$ \\
Macroturbulence & $\zeta_{\mathrm{t}}=3.5 \mathrm{~km} \mathrm{~s}^{-1}$ \\
Microturbulence & $\xi_{\mathrm{t}}=1.8 \mathrm{~km} \mathrm{~s}^{-1}$ \\
\hline
\end{tabular}

were optimized such that the spectra, excluding the "emission bumps" caused by spots, would fit a star with $T_{\mathrm{eff}} \approx 4750 \mathrm{~K}$.

Since our aim was to study the spot activity and not to make an absolute determination of the stellar parameters, it was certainly sufficient to test different sets of parameters and choose the one providing the best fit to an assumed unspotted star. Using the MARCS model atmospheres (Gustafsson et al. 2008), we found that the best starting point was provided by the stellar parameters suggested by Ottmann et al. (1998). The adopted parameters are given in Table 3.

The parameters for the spectral lines were obtained from VALD (Kupka et al. 2000). To optimize the fit to the observations, the $\log g f$-value of some lines was changed. The spectral parameters for the most important lines are given in Table 4 . The full line synthesis included 151 lines.

The need to adjust the spectral parameters could indicate that the element abundances differ from those of the standard MARCS model. It should be emphasized that these kinds of
Table 4. Parameters for important lines ${ }^{c}$.

\begin{tabular}{lccc}
\hline \hline Element ion & $\begin{array}{c}\lambda_{\text {centr }} \\
(\AA)\end{array}$ & $\begin{array}{c}\chi_{\text {low }} \\
(\mathrm{eV})\end{array}$ & $\log (g f)$ \\
\hline $\mathrm{Ni}$ I & 5392.327 & 4.154 & -1.320 \\
$\mathrm{Fe}_{\text {I }}$ & 5393.167 & 3.241 & $-\mathbf{0 . 8 6 5}$ \\
$\mathrm{Co}_{\text {I }}$ & 5393.739 & 4.058 & -0.326 \\
$\mathrm{Fe}_{\text {I }}$ & 5394.346 & 4.835 & -2.102 \\
$\mathrm{Mn}_{\mathrm{I}}$ & 5394.677 & 0.000 & $\mathbf{- 2 . 8 0 3}$ \\
$\mathrm{Fe}_{\text {I }}$ & 5394.680 & 4.186 & $\mathbf{- 1 . 3 2 0}$ \\
$\mathrm{Co}_{\text {I }}$ & 5524.985 & 4.113 & -0.533 \\
$\mathrm{Fe}_{\text {II }}$ & 5525.125 & 3.267 & -4.102 \\
$\mathrm{Fe}_{\text {I }}$ & 5525.477 & 4.209 & -1.994 \\
$\mathrm{Fe}_{\text {I }}$ & 5525.539 & 4.230 & $-\mathbf{1 . 4 8 4}$ \\
$\mathrm{Fe}_{\text {I }}$ & 5525.848 & 5.106 & -1.574 \\
$\mathrm{Sc}_{\text {II }}$ & 5526.790 & 1.768 & $\mathbf{- 0 . 0 1 0}$ \\
$\mathrm{Fe}_{\text {I }}$ & 5633.946 & 4.991 & $\mathbf{- 0 . 4 0 0}$ \\
\hline
\end{tabular}

Notes. ${ }^{(c)}$ Central wavelength, lower excitation potential, and adopted $\log g f$-values. The adjusted values are marked with a bold font.

corrections are necessary in Doppler imaging to reduce the systematic errors caused by discrepancies between the synthetic spectra and observations.

\subsection{Inversion procedure}

A table of line profiles was calculated using MARCS planeparallel atmosphere models with $T_{\text {eff }}$ ranging from $3200 \mathrm{~K}$ to $6000 \mathrm{~K}$ and the stellar parameters listed in Table 3. The local 
line profiles table and the observations were used as input to the inversion code.

The inversion was based on Tikhonov regularization. A regularization parameter of $\Lambda=1 \times 10^{-9}$ and a grid of $80 \times 40$ surface elements were used. The aim was to achieve a difference between the model and the observations of about the same level as the observational noise, i.e. the inverse of the $\mathrm{S} / \mathrm{N}$. This level of convergence was reached after 40 iterations. The deviations are listed in Table 1 . In most cases, the deviation was significantly larger than the inverse of the $\mathrm{S} / \mathrm{N}$-value. This may be caused by systematic errors, e.g. slight shifts in the continuum level or modelling errors. In some seasons, there may also be changes in the spot configuration happening on a timescale shorter than a week. The resulting maps are displayed in Fig. 1 and comparisons between the modelled and observed spectra are shown in Fig. 2.

To make a more reliable comparison with our earlier Doppler images of 1994-2002 (Lindborg et al. 2011), we recalculated the images from 2002 using the new stellar parameters and model atmospheres. Since the earlier observations did not include the same wavelength regions as the present ones, we used the same wavelength regions as in the previous study. The resulting maps are shown in the two uppermost left panels of Fig. 1.

\section{Results}

Our 14 Doppler images of II Peg for the years 2002-2010 are shown in Fig. 1; the color scale of these images is uniform for all the maps, to facilitate the comparison of the strength of the spot activity between the different seasons. Our maps show a continuous evolution of the spot configuration. The temperature of the coolest spot structure is usually about $3500-4000 \mathrm{~K}$, except for July 2004, when the spot activity is quite weak. In December 2004, a clear structure with two spots centred at phases 0.5 and 0.6 has emerged, but during this observational season only three phases were observed. In the July 2005, November 2005, and September 2006 images, two or three larger spot structures can be seen, the longitude of the spots varying strongly in time. During these observing runs, the phase coverage was considerably better than for December 2004. In December 2006, July 2007, and November 2007, the spot activity is again weaker. In September 2008 and December 2009, the Doppler images are dominated by one or two very cool spots, while the spot activity seems to be low for the two observing seasons of December 2008 and August 2009. Furthermore, we note that the deviation between the solution and observations, relative to the noise of the observations, is slightly larger for the December 2008, August 2009, and December 2009 images. This may indicate that the spot activity has evolved rapidly during these seasons.

To study the evolution of the longitudinal spot distribution over time, we calculated average temperatures over all latitudes for each image for both our previous Doppler images (Lindborg et al. 2011) and the present ones, represented in the left and right panels of Fig. 3. In our previous study of II Peg, we detected a longitudinal drift of the spot activity in the orbital rotation frame, which is clearly visible in this plot and also in the maps of Lindborg et al. (2011). This drift is particularly evident during the years 1997-1999. It is hard to detect any such drift in the present images, especially during 2004-2007, when the spots appear to form at more or less random phases. During 2008-2010, however, the main spots persistently occur on one half, roughly between phases 0.5 and 0.8 , of the star.

We also performed a similar analysis by averaging the temperature over longitudes for each latitude. Such a figure could reveal any analogues to the solar butterfly diagram. However, as can be seen in Fig. 4, no clear temporal evolution in the spot latitudes can be detected. The spots are usually concentrated at latitudes $40-80^{\circ}$. However, this kind of analysis requires a consistently satisfactory phase coverage, which is not the case for all the present observations.

We note that the Doppler images from December 2004, November 2005, and September 2008 are based on only 3-5 observed phases. The longitude of a spot can, in principle, be deduced even from a single spectrum, if the spot happens to be near to the centre line of the visible stellar disc. However, the latitude is determined mainly from the amplitude of the radial velocity of the "bump", the measurement of which would require acquisition of more spectra.

Photometric observations can be used as an additional check of the reliability of Doppler images. For this purpose, we used the light curve amplitude and epochs of photometric minima derived from $V$-photometry using the continuous period search method (CPS, Lehtinen et al. 2011). The analysis was applied to $V$-photometry obtained with the T3 0.4 APT at Fairborn Observatory (Arizona, USA). In the CPS-method, a sliding window is used to derive continuous estimates of the light curve parameters. Here we only use the independent measurements of the amplitude and primary photometric minima, i.e. measurements based on non-overlapping data. The full analysis is described by Jetsu et al. (in prep.) and the data are the same as in the study by Roettenbacher et al. (2011).

In Fig. 3, we plot both the temperature averaged over latitude of the Doppler images and the photometric minima. A basic test of the reliability of a Doppler image, is that the longitudes of the main spots, taking into account their combined effects, coincide with the phases of the photometric minima. In the images of December 2004 and November 2005, no major spot structures are seen near to the minima derived from near-simultaneous photometry. The situation with the September 2008 image is somewhat more complicated. In the spectra, there is a clear sign, in the form of a bump, of a spot centred near the rotation phase 0.6 (Fig. 2). This bump causes the large cool spot at phase 0.6 in the Doppler image (Fig. 1). However, in the nearly simultaneous MOST observations of II Peg the main photometric minimum is near the phase 0.8 (Siwak et al. 2010). This is near the location of the secondary spot in our Doppler image. We can thus conclude that some of the major spot concentrations were misplaced or missed in the images from December 2004, November 2005, and September 2008 because of insufficient phase coverage.

Even when the phase coverage is good, one should always be cautious not to over-interpret details of Doppler images. In all our images, we can identify features that may be artifacts typical of Doppler imaging, namely alternating cool and hot regions, arches, and extensions of spots.

The recalculated temperature maps for 2002 (the first two images in Fig. 1) are very similar to those in our previous study (Lindborg et al. 2011), the main difference being that the average temperature is now $\sim 100-150 \mathrm{~K}$ higher. This is a natural consequence of the revised stellar parameters in this study. In addition, there are small shifts in the latitudes of the spot structures, although the longitudes coincide very well. The latter is verified by a comparison of the two last stripes of the left panel with the two first stripes of the right panel in Fig. 3. We conclude that the temperature maps of the present study can be compared to those in our previous Doppler images of 1994-2002, but a shift of $\sim 100-150 \mathrm{~K}$ in the average temperature should be taken into account. 
T. Hackman et al.: Doppler images of II Pegasi for 2004-2010
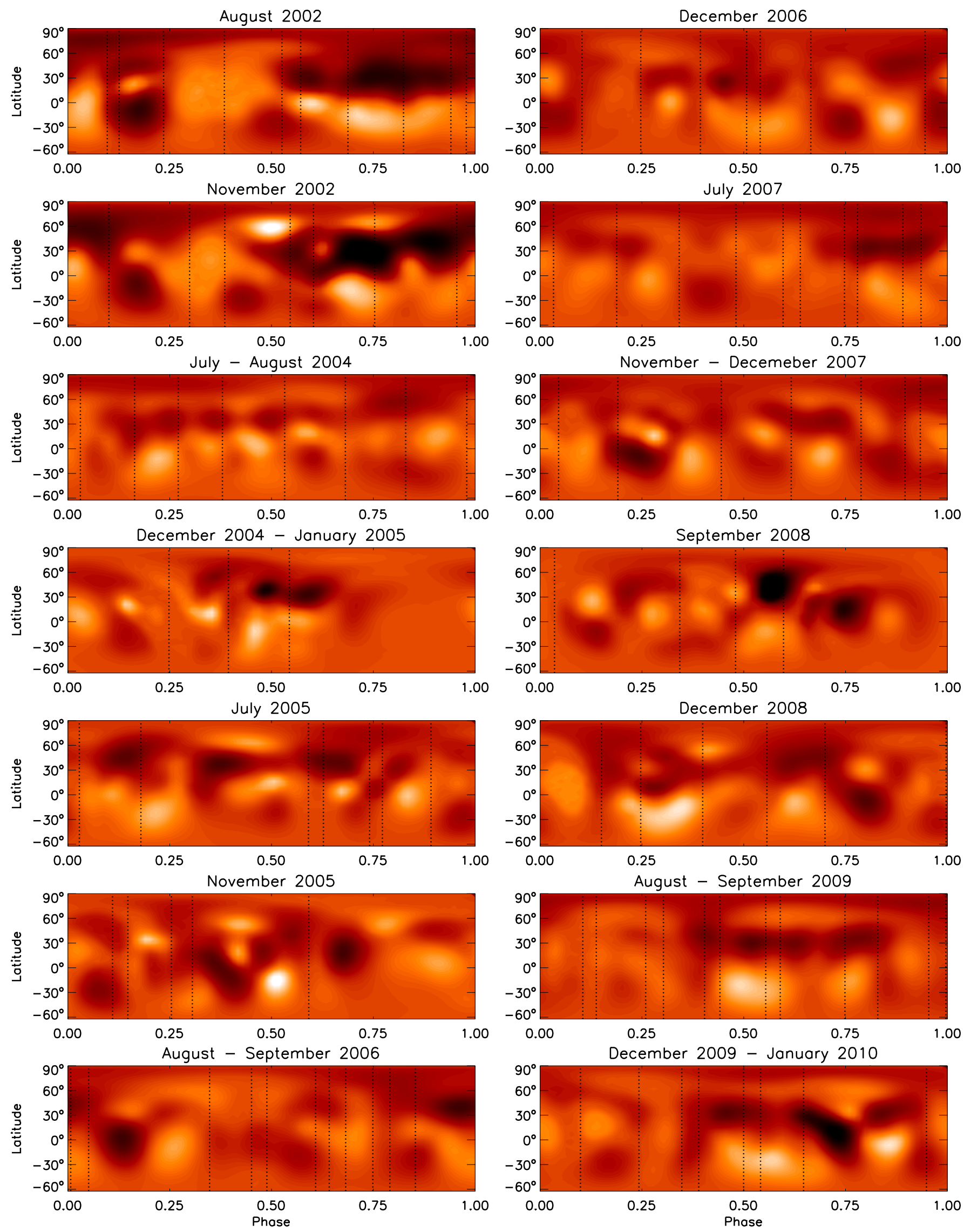

Fig. 1. Doppler imaging temperature maps from 2002-2010 in cylindrical projections. The stellar longitude is given as rotation phase and latitude in degrees. The phases of the observations are marked with vertical dashed lines. 
A\&A 538, A126 (2012)
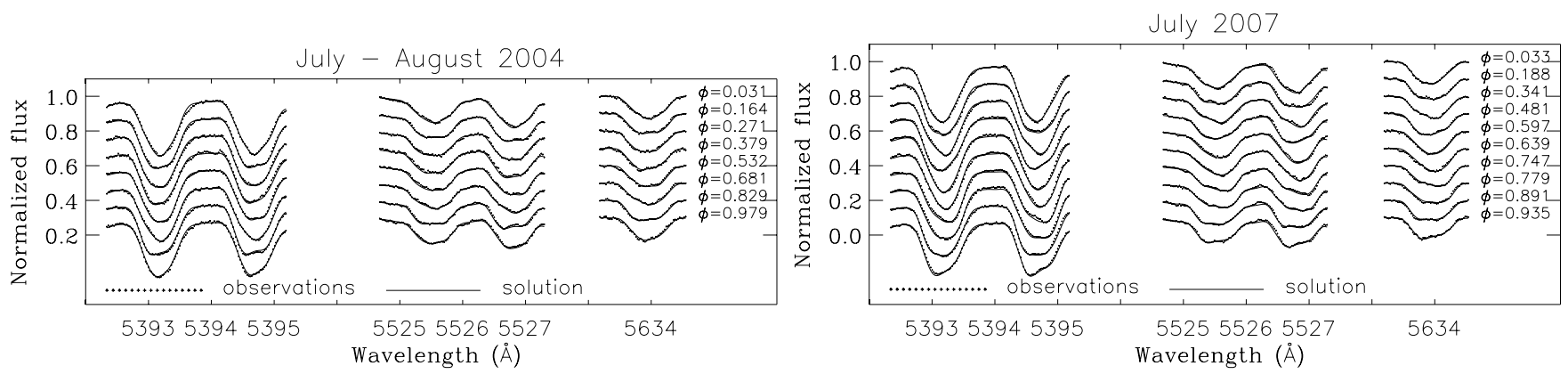

November - Decemeber 2007
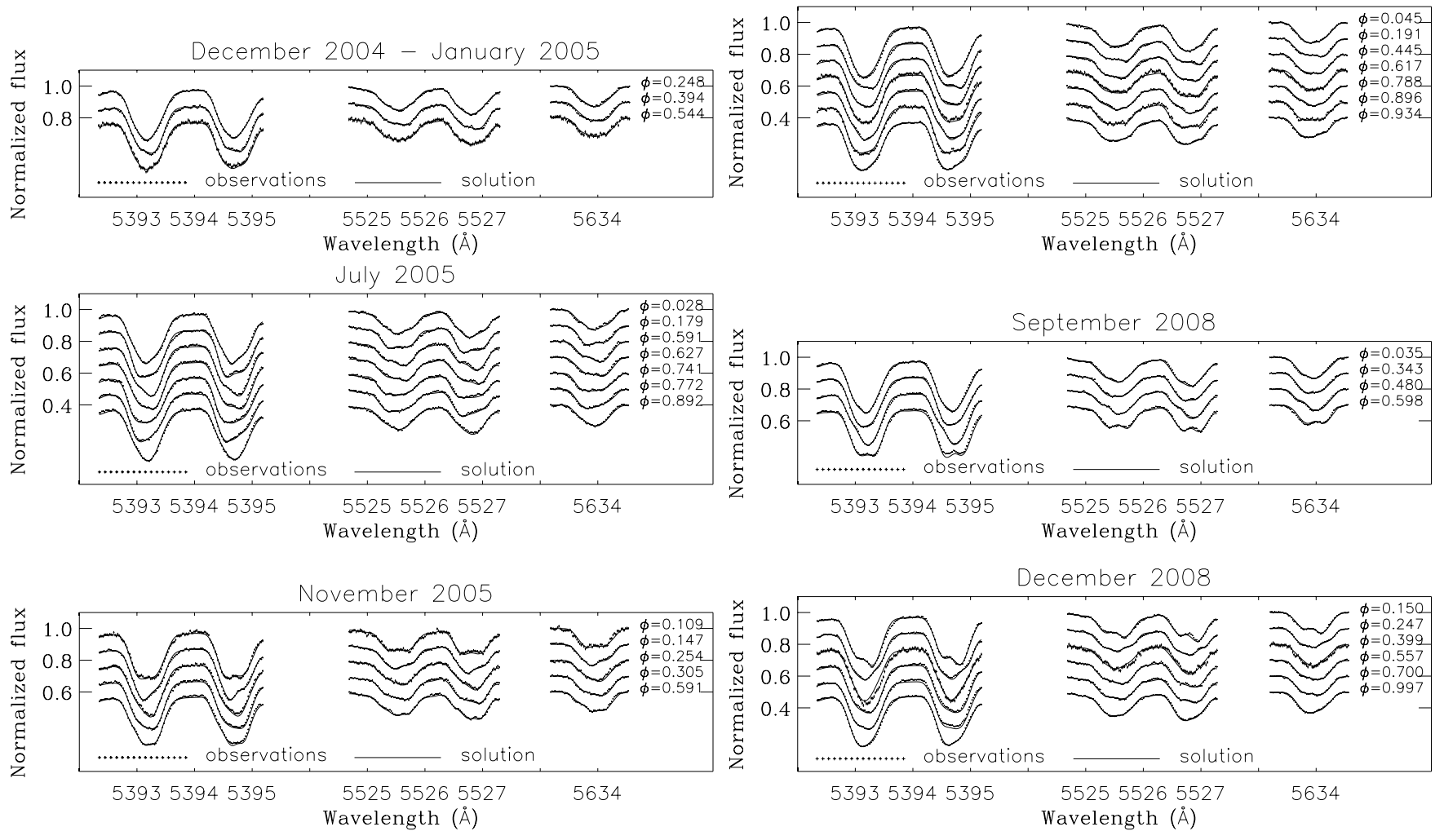

August - September 2006
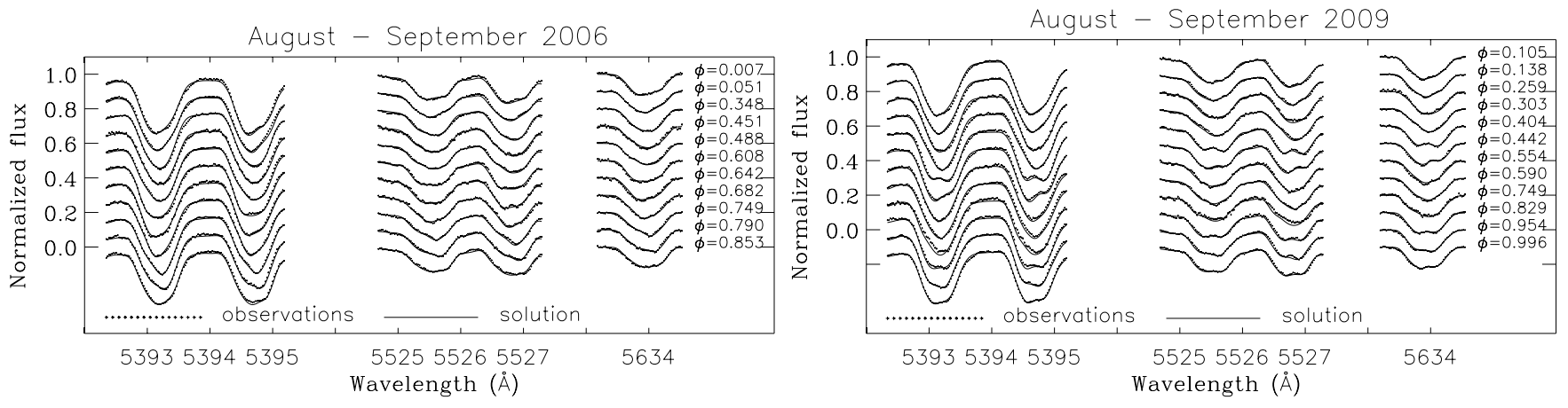

December 2006
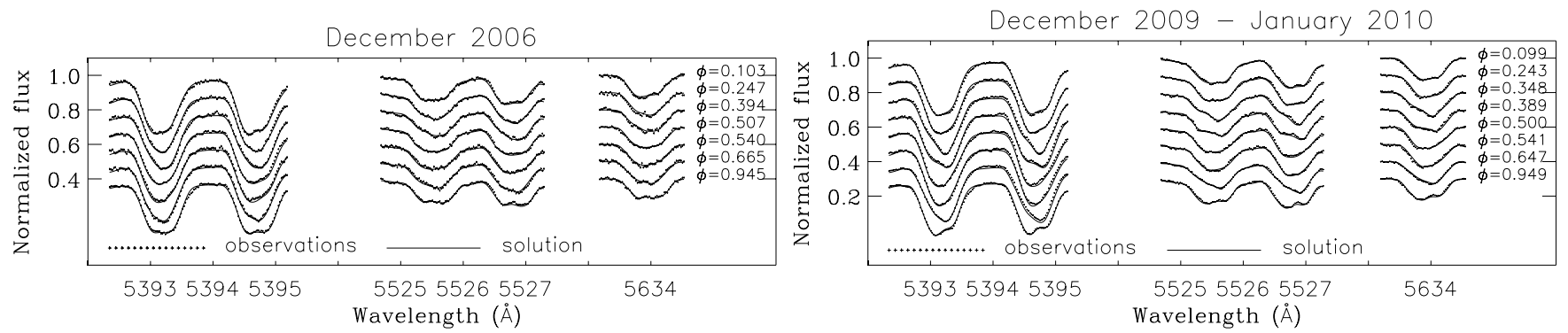

Fig. 2. The spectral observations and Doppler imaging solutions for 2004-2010. 

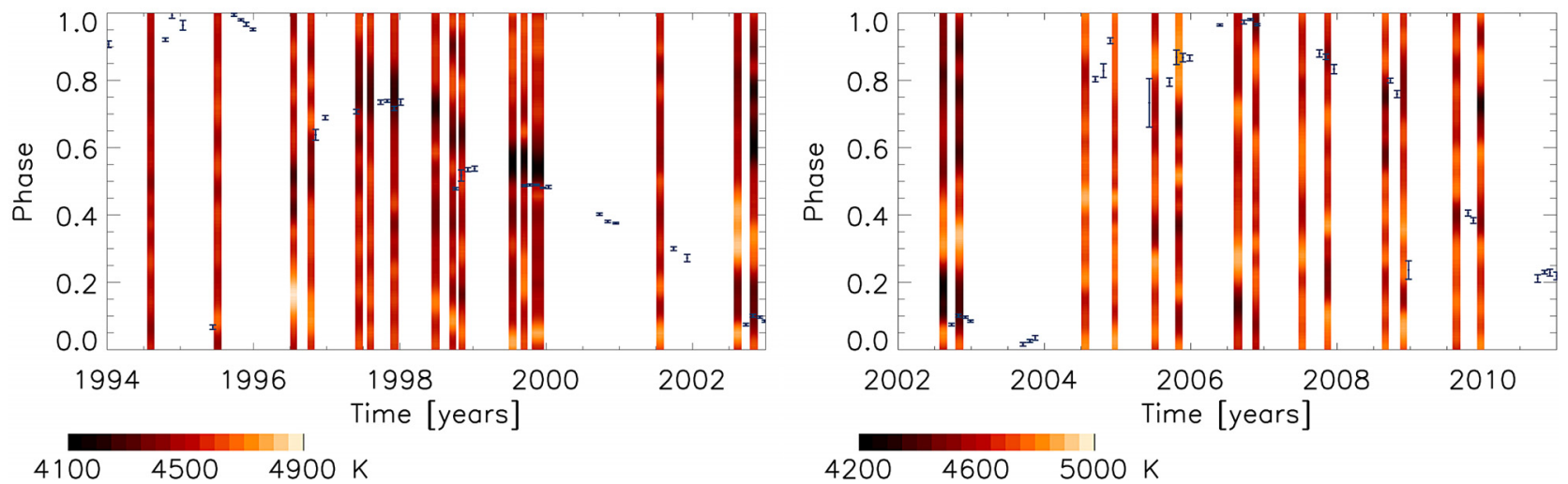

Fig. 3. The longitudinal spot distribution for the years 1994-2002, derived from the old Doppler images (Lindborg et al. 2011), and 2002-2010, derived from the images of the present analysis. Each stripe represents the temperature averaged over all latitudes of the Doppler images. The points with errorbars mark the photometric minima.
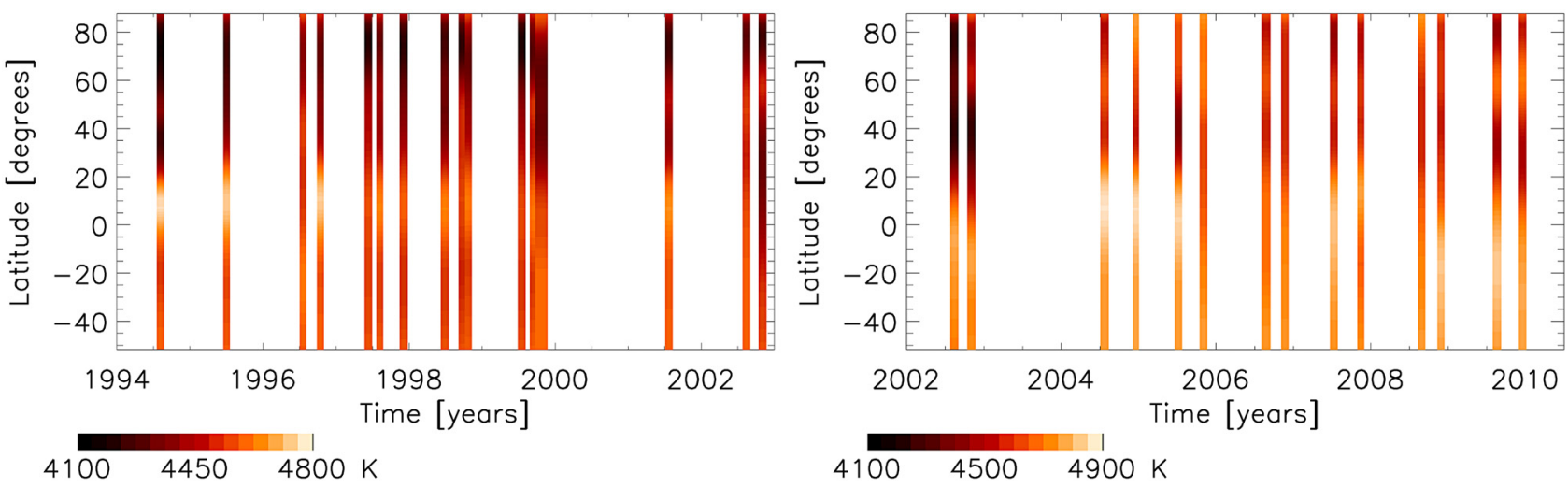

Fig. 4. The latitudinal spot distribution for the years 1994-2002 and 2004-2010. Each stripe represents the temperature averaged over all longitudes of the Doppler images.

The mean temperature of the new images for 2002 is still 50 $100 \mathrm{~K}$ lower than that of the images from 2004-2010 (Fig. 5). This could in principle be a bias caused by the change in the spectroscopic setup. The temperature contrast of Doppler images may be affected by the selection of spectral lines used in the inversion (see e.g. Järvinen \& Berdyugina 2010). However, a natural explanation would be that the spot activity has decreased after 2002. This is confirmed by the small amplitude of the photometric modulation apparent in Fig. 1 of Roettenbacher et al. (2011) at around 2004-2005 (MJD 53 000-53 500), while it was relatively large at around 2000 (MJD 51800 ).

To quantify the level of activity, we defined all surface elements cooler than $T_{\text {spot }}=4200 \mathrm{~K}$ as spots. The spot coverage of each Doppler image was then estimated by calculating the percentage of the surface covered by elements with temperatures lower than $T_{\text {spot }}$. There is a clear decrease in the spot coverage, which explains the increase in the mean temperature occurring after 2002 (Fig. 5). We note that the photometric amplitudes derived by Jetsu et al. (in prep.) also support the notion that the spot activity has decreased after 2002. A comparison with our earlier Doppler images (Lindborg et al. 2011) also shows that the spots were more dominant in the images from 1994-2002 than during 2004-2010.

\section{Conclusions}

In a recent paper (Lindborg et al. 2011), we published 16 temperature maps for the star II Peg during 1994-2002, revealing
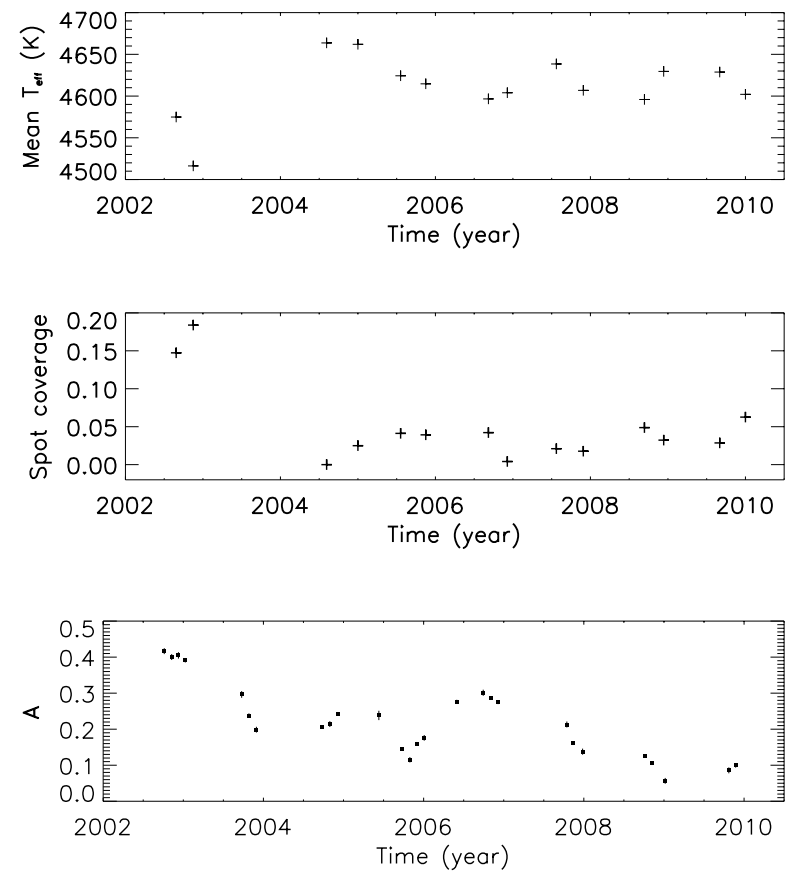

Fig. 5. The mean $T_{\text {eff }}$ and spot coverage of the Doppler imaging maps, and the photometric amplitude 2002-2010.

short-term, irregular, "flip-flop"-type events and a systematic drift of the active regions in the orbital reference frame of the 
binary system. The 12 new temperature maps for 2004-2010 clearly show that the behaviour of the star is quite different from the earlier epoch:

1. We find that the spot activity is generally lower than during 1994-2002. Epochs of low activity, e.g. July-August 2004, December 2006, July 2007, December 2008, and AugustSeptember 2009, alternate with states of higher activity.

2. We are not able to detect any systematic drift of the active regions from the Doppler images with respect to the orbital rotation frame. Instead, consecutive images show far less of a resemblance than during the previous observation period 1994-2002, making it hard to trace any drifts in the spot structures.

3. There is no evidence of a "flip-flop" behaviour. Especially during 2004-2007, the spot distribution over longitudes is more or less random. During 2008-2010, large spots persistently occur between the phases 0.5 and 0.8 , resembling the behaviour seen on the object during earlier epochs.

Several studies have found cyclic behaviour in II Peg. Rodonò et al. (2000) analysed 25 years of photometry and reported cycles of 13.5, 9.5, and 6.8 years in the spot activity. Furthermore, Berdyugina et al. (1999) reported a 4.65 year cycle in the "flipflop" events. We cannot confirm any regular flip-flops. The same conclusion was also drawn in the analysis of photometry by Roettenbacher et al. (2011).

We also see no evidence of a drift in the spot-generating mechanism during 2004-2010 in the Doppler images. However, indications of a drift can be seen in the photometric minima during 2006-2010. We interpret the drift itself as a dynamo wave migrating in the azimuthal direction. The spots are thus generated by an underlying structure with a higher angular velocity than the surface of the star.

It is clear that the star has entered a state of weaker activity than during 1994-2002. The spot evolution seems fast and random, which could mean that a dynamo wave cannot be clearly detected. This could be related to a minimum in the star's cycle. In this respect, the 13.5 year cycle found by Rodonò et al. (2000) is plausible. A period of higher activity and a clearly detectable drifting active longitude, alternating with a period of lower activity, could then constitute the activity cycle. The collected time series of Doppler images, however, is still too short to make a decisive conclusion about the existence of such a stellar cycle.

Acknowledgements. Ilkka Tuominen sadly passed away on March 19, 2011. We wish to express our respect for his importance for the research on magnetically active stars. The work of T.H. was financed by the research programme "Active Suns" at the University of Helsinki. The work of M.L. was supported by the Academy of Finland project 141017, and she has benefited from the NOT research studentship programme. M.J.M. acknowledges the support from the Academy of Finland through the project 218159. O.K. is a Royal Swedish Academy of Sciences Research Fellow supported by grants from the Knut and Alice Wallenberg Foundation and from the Swedish Research Council. We thank the referee for valuable suggestions, which helped to improve the paper.

\section{References}

Berdyugina, S. V., \& Tuominen, I. 1998, A\&A, 336, L25

Berdyugina, S. V., Berdyugin, A. V., Ilyin, I., \& Tuominen, I. 1998a, A\&A, 340, 437

Berdyugina, S. V., Jankov, S., Ilyin, I., Tuominen, I., \& Fekel, F. C. 1998b, A\&A, 334,863

Berdyugina, S. V., Berdyugin, A. V., Ilyin, I., \& Tuominen, I. 1999, A\&A, 350, 626

Doyle, J. G., Butler, C. J., Byrne, P. B., et al. 1989, A\&A, 223, 219

Frasca, A., Biazzo, K., Taş, G., Evren, S., \& Lanzafame, A. C. 2008, A\&A, 479, 557

Frasca, A., Fröhlich, H.-E., Bonanno, A., et al. 2011, A\&A, 532, A81

Gustafsson, B., Edvardsson, B., Eriksson, K., et al. 2008, A\&A, 486, 951

Hackman, T., Jetsu, L., \& Tuominen, I. 2001, A\&A, 374, 171

Ilyin, I. V. 2000, Ph.D. Thesis, University of Oulu

Järvinen, S. P., \& Berdyugina, S. V. 2010, A\&A, 521, A86

Jeffers, S. V., \& Donati, J.-F. 2008, MNRAS, 390, 635

Kitchatinov, L. L., \& Rüdiger, G. 1999, A\&A, 344, 911

Korhonen, H., \& Elstner, D. 2011, A\&A, 532, A106

Krause, F., \& Rädler, K.-H. 1980, Mean-field magnetohydrodynamics and dynamo theory (Oxford: Pergamon Press)

Kupka, F. G., Ryabchikova, T. A., Piskunov, N. E., Stempels, H. C., \& Weiss, W. W. 2000, Baltic Astron., 9, 590

Lehtinen, J., Jetsu, L., Hackman, T., Kajatkari, P., \& Henry, G. W. 2011, A\&A, 527, A136

Lindborg, M., Korpi, M. J., Hackman, T., et al. 2011, A\&A, 526, A44

Makarov, V. V. 2003, AJ, 126, 1996

Mohin, S., \& Raveendran, A. V. 1993, A\&A, 277, 155

Moss, D., Barker, D. M., Brandenburg, A., \& Tuominen, I. 1995, A\&A, 294, 155

Ottmann, R., Pfeiffer, M. J., \& Gehren, T. 1998, A\&A, 338, 661

Piskunov, N. E. 1991, in The Sun and Cool Stars, Activity, Magnetism, Dynamos, ed. I. Tuominen, D. Moss, \& G. Rüdiger (Heidelberg: SpringerVerlag), Lecture Notes in Physics, 380, IAU Colloq., 130, 309

Rodonò, M., Messina, S., Lanza, A. F., Cutispoto, G., \& Teriaca, L. 2000, A\&A, 358,624

Roettenbacher, R. M., Harmon, R. O., Vutisalchavakul, N., \& Henry, G. W. 2011, AJ, 141, 138

Siwak, M., Rucinski, S. M., Matthews, J. M., et al. 2010, MNRAS, 408, 314

Teriaca, L., \& Catalano, S. 1999, in Solar and Stellar Activity: Similarities and Differences, ed. C. J. Butler, \& J. G. Doyle (San Francisco: ASP), ASP Conf. Ser., 158, 243

Tuominen, I., Berdyugina, S., \& Korpi, M. 2002, Astron. Nachr., 323, 367

Vogt, S. S., Penrod, G. D., \& Hatzes, A. P. 1987, ApJ, 321, 496 